\title{
Lack of connection between the uterine cervix and corpus in an adolescent treated in childhood for teratoma of the ovary
}

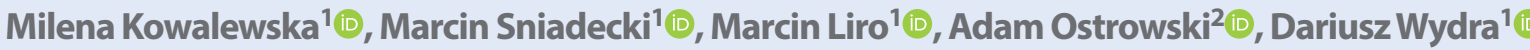 \\ ${ }^{1}$ Department of Gynecology, Gynecologic Oncology and Gynecologic Endocrinology, Medical University of Gdansk, Poland \\ ${ }^{2}$ Department of General and Oncologic Urology, Nicolaus Copernicus University, Bydgoszcz, Poland
}

A 19-year-old female patient was admitted to the Clinic because of primary amenorrhea.

In the initial anamnesis, it was learned that at the age of 10 , the patient had undergone surgery to remove an immature

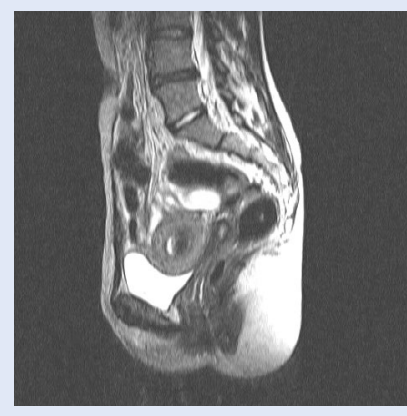

Figure 1. T2-weighted MRI scan showing no connection between the cervical canal and uterine cavity

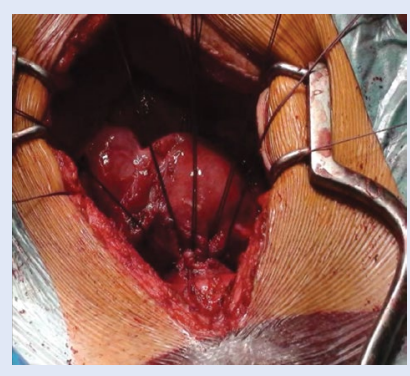

Figure 2. Reconstructed connection between the uterus and cervix

teratoma of the left ovary at another hospital. Following the operation, adjuvant chemotherapy treatment was administered. The patient underwent surgery six years later. During that operation, an serous ovarian cyst of the right ovary was removed.

Given the reason that the patient reported to the clinic, an urography and ultrasound were performed. The result of the former was normal; and the latter indicated grounds for suspecting that the cervix and uterine corpus were not connected.

To either confirm or eliminate this possibility, an MRI of the pelvis was scheduled. That test confirmed the suspected abnormality, indicating the presence of fluid within the uterine cavity possessing a different signal, arranged in horizontal levels. It was confirmed that there seemed to be no connection between the cervical canal and uterine cavity (Fig. 1).

Based on the results of all of the tests, it was decided to operate. After opening the abdominal cavity, it was evident that the uterus and cervix were not connected to each other, nor was the septum between them visible. Thus, during the operation, the connection between the uterus and cervix was reconstructed (Fig. 2).

Again, despite her treatments, the patient reported to the hospital on repeated occasions with severe menstrual bleeding and pain. In addition to standard diagnostics, a psychiatric consultation was commissioned because a somatic symptom disorder was suspected; however, it was not confirmed. After repeated consultations and due to the exhaustion of all therapeutic methods to alleviate the patient's pain, a hysterectomy was ordered. The patient intended to have the procedure performed in another hospital, but she delayed making the final decision because of her young age and the possibility of losing her fertility. After several years, in-depth diagnostics was repeated and a presacral tumor was found, which turned out to be a distant metastasis of mature ovarian teratoma.

\section{DISCUSSION}

So far, two cases of this defect have been described, however they might be of a different origin [1, 2]. It is very unlikely for such a defect to occur, because the uterus develops ontogenetically from paired ducts (the Müllerian ducts). As the ducts connect at the sagittal plane, the developmental defects described by us at the beginning concern both sides of the uterus. Thus, the formation of a separate corpus and cervix is unlikely. The author of the paper concerning a similar defect believes, that the formation of uterus is bi-directional and the development of uterine corpus and cervix is possible without the middle component [2]. However, both cases described in the literature differ from our patient. In the above cases, connective tissue was found between uterine corpus and cervix, whereas it has not been observed in our patient. Moreover, our patient had an abdominal surgery in her childhood.

Due to the fact that the patient was not diagnosed with a malformation, it was suggested that the cause was iatrogenic. However, there was no confirmation of that assumption in the medical documentation from another hospital. It is probable that, although the presence of this defect was not confirmed during previous operations, it had already been present. However, the internal reproductive organs in young girls are quite small and more attention was paid to removal of neoplasm from the genital organs.

\section{REFERENCES}

1. Kisu I, Tanaka K, Banno K, et al. Repair of congenital ,disconnected uterus': a new female genital anomaly? Hum Reprod. 2015; 30(1): 46-48, doi: 10.1093/humrep/deu287, indexed in Pubmed: 25376460.

2. Beran BD, Pereira LF, Zimberg S, et al. Primary Amenorrhea and Endometriosis Secondary to an Unusual Müllerian Anomaly. J Minim Invasive Gynecol. 2018; 25(2): 318-319, doi: 10.1016/j.jmig.2017.04.011, indexed in Pubmed: 28461177. 